\title{
SINGULARITIES OF BLASCHKE NORMAL MAPS OF CONVEX SURFACES
}

\author{
KENTARO SAJI, MASAAKI UMEHARA, AND KOTARO YAMADA
}

\begin{abstract}
We prove that the difference between the numbers of positive swallowtails and negative swallowtails of the Blaschke normal map for a given convex surface in affine space is equal to the Euler number of the subset where the affine shape operator has negative determinant.
\end{abstract}

\section{INTRODUCTION.}

Throughout this Note, we assume that $M^{2}$ is a compact oriented 2-manifold without boundary. Let $\varphi$ be a bundle homomorphism of the tangent bundle $T M^{2}$ into a vector bundle $E$ of rank 2 over $M^{2}$. A point $p$ on $M^{2}$ is called a singular point if the linear map $\varphi_{p}: T_{p} M \rightarrow E_{p}$ is not bijective. We denote by $\Sigma_{\varphi}$ the set of singular points of $\varphi$. We assume that $E$ is orientable, that is, there is a non-vanishing section $\mu: M^{2} \rightarrow E^{*} \wedge E^{*}$, where $E^{*}$ is the dual vector bundle of $E$. We now fix a metric $\langle$,$\rangle on E$. Multiplying a suitable $C^{\infty}$-function on $M^{2}$, we may assume that $\mu\left(e_{1}, e_{2}\right)=1$ holds for any oriented orthonormal frame $e_{1}, e_{2}$ on $E$. By using a positively oriented local coordinate system $(U ; u, v)$, the signed area form $d \hat{A}$, the signed area density function $\lambda$, and the (un-signed) area form $d A$ are defined by

$$
d \hat{A}:=\varphi^{*} \mu=\lambda d u \wedge d v, \quad d A:=|\lambda| d u \wedge d v .
$$

Both $d \hat{A}$ and $d A$ are independent of the choice of $(u, v)$, and are 2-forms globally defined on $M^{2}$. When $\varphi$ has no singular points, these two forms coincide up to sign. We set

$$
M^{+}:=\left\{p \in M^{2} \backslash \Sigma_{\varphi} ; d \hat{A}_{p}=d A_{p}\right\}, \quad M^{-}:=\left\{p \in M^{2} \backslash \Sigma_{\varphi} ; d \hat{A}_{p}=-d A_{p}\right\} .
$$

The singular set $\Sigma_{\varphi}$ coincides with $\partial M^{+}=\partial M^{-}$. A singular point $p\left(\in \Sigma_{\varphi}\right)$ on $M^{2}$ is called non-degenerate if the derivative $d \lambda$ does not vanish at $p$. In a neighborhood of a non-degenerate singular point, the singular set can be parametrized as a regular curve $\gamma(t)$ on $M^{2}$, called the singular curve. The tangential direction of $\gamma$ is called the singular direction. The direction of the kernel of $\varphi_{p}$ is called the null direction, which is one dimensional. There exists a smooth non-vanishing vector field $\eta(t)$ along $\gamma$ pointing in the null direction, called the null vector field.

Definition 1.1. Take a non-degenerate singular point $p \in M^{2}$ and let $\gamma(t)$ be the singular curve satisfying $\gamma(0)=p$. Then $p$ is called an $A_{2}$-point if the null direction $\eta(0)$ is transversal to the singular direction $\dot{\gamma}(0)=d \gamma /\left.d t\right|_{t=0}$. If $p$ is not an $A_{2}$-point, but satisfies that $d(\dot{\gamma}(t) \wedge \eta(t)) / d t$ does not vanish at $p=\gamma(0)$, it is called an $A_{3}$-point, where $\wedge$ is the exterior product on $T M^{2}$. We fix an $A_{3}$-point

2000 Mathematics Subject Classification. 53A15, 53C45, 57R45. 
$p$. If the angle of the region $M^{-}$(resp. $M^{+}$) at $p$ with respect to the pull-back metric $d s^{2}:=\varphi^{*}\langle$,$\rangle is zero, then it is called a positive (resp. negative) A_{3}$-point. $\left(A_{3}\right.$-points are either positive or negative, see [6]).

We now fix a metric connection $D$ of $(E,\langle\rangle$,$) . Let \gamma(t)$ be a regular curve on $M^{2}$ consisting only of $A_{2}$-points. Take a null vector field $\eta(t)$ such that $(\dot{\gamma}, \eta)$ is a positive frame of $T M^{2}$ along $\gamma$. Then

$$
\kappa_{s}(t)=\operatorname{sgn}(d \lambda(\eta(t))) \frac{\mu\left(\varphi(\dot{\gamma}(t)), D_{t} \varphi(\dot{\gamma}(t))\right)}{\langle\varphi(\dot{\gamma}(t)), \varphi(\dot{\gamma}(t))\rangle^{3 / 2}}
$$

is called the singular curvature of $\gamma$ at $t$ (see [5] and [6]).

For an oriented orthonormal frame field $e_{1}, e_{2}$ of $E$ defined on $U \subset M^{2}$, there is a unique 1-form $\omega$ on $U$ such that $D_{X} e_{1}=-\omega(X) e_{2}, D_{X} e_{2}=\omega(X) e_{1}$. Then $d \omega$ does not depend on the choice of $e_{1}, e_{2}$, and there is a $C^{\infty}$-function $K_{\varphi, D}$ on $M^{2} \backslash \Sigma_{\varphi}$ such that

$$
d \omega=K_{\varphi, D} d \hat{A} .
$$

We call $K_{\varphi, D}$ the Gaussian curvature of $D$ with respect to $\varphi$. Let $\bar{D}$ be the pull-back of $D$ on $M^{2} \backslash \Sigma_{\varphi}$. Let $\sigma(t)$ be a regular curve on $U \backslash \Sigma_{\varphi}$ with the arclength parameter $t$ with respect to $d s^{2}=\varphi^{*}\langle$,$\rangle . We take a unit normal vector n(t)$ such that $(\dot{\sigma}, n)$ gives a positive frame on $T M^{2}$. On the other hand, we take $\hat{n}(t) \in E$ such that $(\varphi(\dot{\sigma}), \hat{n})$ gives a positive frame on $E$. We can define two geodesic curvatures;

$$
\kappa_{g}=d s^{2}\left(\bar{D}_{t} \dot{\sigma}(t), n(t)\right), \quad \hat{\kappa}_{g}=\left\langle D_{t} \varphi(\dot{\sigma}(t)), \hat{n}(t)\right\rangle .
$$

Here, $\hat{\kappa}_{g}(t)$ is well-defined even when $\sigma(t)$ passes through the set $\Sigma_{\varphi}$. Since $\varphi(n)=$ $\operatorname{sgn}(\lambda) \hat{n}$, it holds that $\kappa_{g}=\operatorname{sgn}(\lambda) \hat{\kappa}_{g}$. We set $\left(\bar{e}_{1}, \bar{e}_{2}\right)=\left(\varphi^{-1}\left(e_{1}\right), \varphi^{-1}\left(e_{2}\right)\right)$ if $U \subset M^{+}$and set $\left(\bar{e}_{1}, \bar{e}_{2}\right)=\left(\varphi^{-1}\left(e_{2}\right), \varphi^{-1}\left(e_{1}\right)\right)$ if $U \subset M^{-}$. Then $\left(\bar{e}_{1}, \bar{e}_{2}\right)$ gives an oriented orthonormal frame on $T M^{2}$, and there is a $C^{\infty}$-function $\theta=\theta(t)$ such that $\dot{\sigma}=\cos \theta \bar{e}_{1}+\sin \theta \bar{e}_{2}$ and $n=-\sin \theta \bar{e}_{1}+\cos \theta \bar{e}_{2}$. Then we get

$$
\kappa_{g} d t=d \theta-(\operatorname{sgn} \lambda) \omega .
$$

If the connection $D$ satisfies the condition

$$
D_{X} \varphi(Y)-D_{Y} \varphi(X)-\varphi([X, Y])=0
$$

for all vector fields $X, Y$ on $M^{2},(E,\langle\rangle, D,, \varphi)$ is called a coherent tangent bundle. Under the condition (4), $\bar{D}$ gives the Levi-Civita connection of $d s^{2}$ on $M^{2} \backslash \Sigma_{\varphi}$, and $K_{\varphi, D}$ coincides with the usual Gaussian curvature. We consider a contractible triangular domain $\triangle \mathrm{ABC}$ on $M^{2} \backslash \Sigma_{\varphi}$ such that it lies on the left-hand side of the regular arcs $\mathrm{AB}, \mathrm{BC}, \mathrm{CA}$ which meet transversally at $\mathrm{A}, \mathrm{B}, \mathrm{C} \in M^{2}$. By applying the Stokes formula, (2) and (3) yield that

$$
\angle \mathrm{A}+\angle \mathrm{B}+\angle \mathrm{C}-\pi=\int_{\partial \triangle \mathrm{ABC}} \kappa_{g} d \tau+\int_{\triangle \mathrm{ABC}} K_{\varphi, D} d A,
$$

where $\angle A, \angle B, \angle C$ are the interior angles of the domain $\triangle A B C$. To prove this, we do not need to assume that $\bar{D}$ is the Levi-Civita connection. However, we must remember that $K_{\varphi, D}$ is not the usual Gaussian curvature. One crucial point in this setting is that

$$
\int_{M^{2}} K_{\varphi, D} d \hat{A}=\frac{1}{2 \pi} \int_{M^{2}} d \omega
$$


coincides with the Euler characteristic $\chi_{E}$ of the vector bundle E. In [6] (see also $[5])$, the authors gave the following two Gauss-Bonnet type formulas

(6) $\chi_{E}=\chi\left(M^{+}\right)-\chi\left(M^{-}\right)+S_{+}-S_{-}, \quad 2 \pi \chi\left(M^{2}\right)=\int_{M^{2}} K_{\varphi, D} d A+2 \int_{\Sigma_{\varphi}} \kappa_{s} d \tau$,

under the assumption that $(E,\langle\rangle, D,, \varphi)$ is a coherent tangent bundle, where $d \tau$ is the arclength element on the singular set and $S_{+}, S_{-}$are the numbers of positive and negative $A_{3}$-points, respectively. After the publication of [6], the authors found that the proof in [6] is based only on the formula (5) and the identity $\kappa_{g}=\operatorname{sgn}(\lambda) \hat{\kappa}_{g}$. So we can conclude that the two formulas (6) hold without assuming (4). Moreover, we can generalize these two formulas to $\varphi$ admitting more general singularities; in other words, Theorem B in [6] holds on $\varphi$ without assuming (4). If $E=T M^{2}$, then $\chi_{E}$ coincides with $\chi\left(M^{2}\right)=\chi\left(M^{+}\right)+\chi\left(M^{-}\right)$in our setting. So we get the following

Theorem 1.2. Let $\varphi: T M^{2} \rightarrow T M^{2}$ be a bundle homomorphism whose singular set consists only of $A_{2}$ and $A_{3}$-points. Then $2 \chi\left(M^{-}\right)=S_{+}-S_{-}$and $\int_{M^{-}} K_{\varphi, D} d \hat{A}=$ $\int_{\Sigma_{\varphi}} \kappa_{s} d \tau$ hold.

Let $f: M^{2} \rightarrow\left(N^{3}, g\right)$ be an immersion into an orientable Riemannian 3manifold. Then there is a globally defined unit normal vector field $\nu$ along $f$. We define the shape operator $\varphi: T M^{2} \ni v \mapsto-D_{v} \nu \in T M^{2}$, as a bundle homomorphism, where $D$ is the Levi-Civita connection of $\left(N^{3}, g\right)$. A singular point of $\varphi$ is called an inflection point of $f$. We get the following

Corollary 1.3 (A generalization of the Bleeker-Wilson formula). Suppose that the shape operator admits only $A_{2}$ and $A_{3}$-points. Then $2 \chi\left(M^{-}\right)=I_{+}-I_{-}$holds, where $I_{+}$(resp. $\left.I_{-}\right)$is the number of positive (resp. negative) $A_{3}$-inflection points.

The original formula was for the case $N^{3}=\boldsymbol{R}^{3}$ (see [2]). In [7], the authors pointed out that the formula holds for space forms. Also, they gave in [7] several applications of (6) under the assumption (4). However, now we can remove (4), and we get also the results that follow here.

\section{Rotation OF VECTOR FIELDS.}

We fix a Riemannian metric $d s^{2}$ on $M^{2}$. There is a unique 2-form $\mu$ on $M^{2}$ such that $\mu\left(e_{1}, e_{2}\right)=1$ where $e_{1}, e_{2}$ is a local oriented orthonormal frame field on $M^{2}$. Let $X$ be a vector field on $M^{2}$. The $C^{\infty}$-function $\operatorname{rot}(X):=\mu\left(D_{e_{1}} X, D_{e_{2}} X\right)$ defined on $M^{2}$ is called the rotation of $X$, where $D$ is the Levi-Civita connection of $\left(M^{2}, d s^{2}\right)$. Consider a bundle homomorphism $\varphi: T M^{2} \ni v \mapsto D_{v} X \in T M^{2}$. The singular set $\Sigma_{X}$ of $\varphi$ coincides with the zeros of $\operatorname{rot}(X)$, called the set of irrotational points. Moreover, an $A_{3}$-singular point is called an irrotational cusp. In fact, if $M^{2}=\boldsymbol{R}^{2}$ is the Euclidean plane, then $X$ induces a map $\tilde{X}: \boldsymbol{R}^{2} \rightarrow \boldsymbol{R}^{2}$, and $A_{3}$ (resp. $A_{2}$ ) points correspond to cusps (resp. folds) of $\tilde{X}$ (see [7]). Suppose that $X$ admits only $A_{2}$ and $A_{3}$-irrotational points. Then $\Sigma_{X}$ consists of a finite disjoint union of closed regular curves $\gamma_{1}, \ldots, \gamma_{m}$ on $M^{2}$ such that $M^{+}$lies in the left hand side of each $\gamma_{j}$. Then the singular curvature on $\gamma_{j}$ is given by $\kappa_{s}:=\mu(\dot{X}, \ddot{X}) /|\dot{X}|^{3}$ (we propose to call it the irrotational curvature), where $\dot{X}=D_{\dot{\gamma}_{j}(t)} X$ and $\ddot{X}=$ $D_{\dot{\gamma}_{j}(t)} \dot{X}$. The following assertion follows directly from Theorem 1.2. 
Proposition 2.1. Suppose that $X$ admits only $A_{2}$ and $A_{3}$-irrotational points. Then it holds that

$$
\begin{aligned}
& 2 \chi\left(M^{-}\right)=C_{+}-C_{-}, \quad \int_{M^{-}} K_{\varphi, D} d \hat{A}=\int_{\Sigma_{X}} \kappa_{s} d \tau, \\
& M^{-}:=\left\{p \in M^{2} ; \operatorname{rot}(X)_{p}<0\right\},
\end{aligned}
$$

where $C_{+}$(resp. $\left.C_{-}\right)$is the number of positive (resp. negative) irrotational cusps.

\section{Singularities of Blaschke normal maps on CONVEX surfaces.}

Let $S^{2}$ be a 2 -sphere and $f: S^{2} \rightarrow \boldsymbol{R}^{3}$ a strictly convex embedding. In affine differential geometry, it is well-known that there are a transversal vector field $\xi$ along $f$, a torsion free connection $\nabla$, a bundle homomorphism $\alpha: T S^{2} \rightarrow T S^{2}$ (called the affine shape operator), and a positive definite symmetric covariant tensor $h$ such that (cf. [4]) $D_{X} Y=\nabla_{X} Y+h(X, Y) \xi$ and $D_{X} \xi=-\alpha(X)$ for any vector fields $X, Y$ on $S^{2}$, where $D$ is the canonical affine connection on $\boldsymbol{R}^{3}$. Moreover, such a structure $(\xi, \nabla, \alpha, h)$ is uniquely determined up to a constant multiplication of $\xi$. Here $\xi$ induces a map $\tilde{\xi}: S^{2} \rightarrow \boldsymbol{R}^{3}$ called the Blaschke normal map. It is obvious that the singular points of $\alpha$ coincides with those of $\tilde{\xi}$.

Lemma 3.1. The Blaschke normal map $\tilde{\xi}$ is a wave front (cf. [1] for the definition of wave front).

Proof. Consider a non-zero section $L: S^{2} \ni p \mapsto\left(\tilde{\xi}_{p}, \nu_{p}\right) \in T^{*} \boldsymbol{R}^{3}=\boldsymbol{R}^{3} \times\left(\boldsymbol{R}^{3}\right)^{*}$, where $\nu: S^{2} \rightarrow\left(\boldsymbol{R}^{3}\right)^{*}$ is the map into the dual vector space $\left(\boldsymbol{R}^{3}\right)^{*}$ of $\boldsymbol{R}^{3}$ such that $\nu_{p}\left(\tilde{\xi}_{p}\right)=1$ and $\nu_{p}\left(d f\left(T_{p} S^{2}\right)\right)=\{0\}$ for each $p \in S^{2}$. Take a local coordinate system $\left(u_{1}, u_{2}\right)$ of $S^{2}$. Then we have that

$$
\begin{aligned}
\nu_{u_{i}}\left(f_{u_{j}}\right)=D_{\partial_{i}} \nu\left(f_{u_{j}}\right)= & \nu\left(D_{\partial_{i}} f_{u_{j}}\right) \\
& =-\nu\left(\nabla_{\partial_{i}} \partial_{j}+h\left(\partial_{i}, \partial_{j}\right) \tilde{\xi}\right)=-h\left(\partial_{i}, \partial_{j}\right) \quad(i, j=1,2),
\end{aligned}
$$

where $\partial_{i}:=\partial / \partial u_{i}$ and $f_{u_{i}}:=d f\left(\partial_{i}\right)$. Since $h$ is positive definite, $\nu_{u_{1}}, \nu_{u_{2}}$ are linearly independent. Moreover, $\nu, \nu_{u_{1}}, \nu_{u_{2}}$ are also linearly independent, since $\nu\left(d f\left(T_{p} S^{2}\right)\right)=0$. In particular, $L$ induces a Legendrian immersion of $S^{2}$ into the projective cotangent bundle $P\left(T^{*} \boldsymbol{R}^{3}\right)$ of $T^{*} \boldsymbol{R}^{3}$.

By applying the criteria of cuspidal edges and swallowtails (cf. [7], $A_{2}$ and $A_{3}$ points correspond to the cuspidal edges and swallowtails of the Blaschke normal map $\tilde{\xi}$. So we get the following

Theorem 3.2. Suppose that $\tilde{\xi}$ admits only cuspidal edges and swallowtails. Then $2 \chi\left(M^{-}\right)=S_{+}-S_{-}$holds, where $M^{-}:=\left\{p \in S^{2} ; \operatorname{det}(\alpha(p))<0\right\}$ and $S_{+}$(resp. $S_{-}$) is the number of positive (resp. negative) swallowtails of $\tilde{\xi}$.

A different formula for $S_{+}+S_{-}$is given by Izumiya-Marer [3].

\section{REFERENCES}

[1] V. I. Arnol'd, S. M. Gusein-Zade and A. N. Varchenko, Singularities of Differentiable MAPS, Vol. 1, Monographs in Math. 82, Birkhäuser (1985).

[2] D. Bleecker and L. Wilson, Stability of Gauss maps, Illinois J. of Math. 22 (1978), 279-289. 
[3] S. Izumiya, W. I. Marar, The Euler characteristic of a general wavefront in a 3-manifold, Proc. Amer. Math. Soc. 118 (1993) 1347-1350.

[4] K. Nomizu, and T. Sasaki, Affine Differential Geometry, Cambridge University Press, Cambridge (1994).

[5] K. Saji, M. Umehara and K. Yamada, The geometry of fronts, Ann. of Math. 169 (2009), $491-529$

[6] K. Saji, M. Umehara and K. Yamada, Behavior of corank one singular points on wave fronts, Kyushu J. Math. 62 (2008), 259-280.

[7] K. Saji, M. Umehara and K. Yamada, The intrinsic duality of wave fronts, preprint, arXiv:0910.3456.

E-mail address: ksaji@gifu-u.ac.jp

E-mail address: umehara@math.sci.osaka-u.ac.jp

E-mail address: kotaro@math.titech.ac.jp

(saji) Department of Mathematics, Faculty of Education, Gifu University, Yanagido 1-1, GiFu 501-1193, JAPAN

(umehara) Department of Mathematics, Graduate School of Science, Osaka UniverSiTy, TOYONAKa, OSAKa 560-0043, JAPAN

(yamada) Department of Mathematics, Tokyo Institute of Technology, O-okayama, MEguro, TOKYO 152-8551, JAPAN 